\title{
Storage Ring EDM Experiments
}

\author{
Yannis K. Semertzidis ${ }^{1,2, a}$ \\ ${ }^{1}$ Center for Axion and Precision Physics Research, IBS, Daejeon 305-701, Republic of Korea \\ ${ }^{2}$ Dept. of Physics, KAIST, Daejeon 305-701, Republic of Korea
}

\begin{abstract}
Dedicated storage ring electric dipole moment (EDM) methods show great promise advancing the sensitivity level by a couple orders of magnitude over currently planned hadronic EDM experiments. We describe the present status and recent updates of the field.
\end{abstract}

\section{Introduction}

Electric dipole moments of fundamental particles violate separately the discrete symmetries of parity $(\mathrm{P})$ and time (T) reversal. Assuming exact CPT symmetry, where (C) denotes the symmetry with charge, T-symmetry violation also means CP-violation. $\mathrm{CP}$-violation is one of Sakharov's necessary conditions to develop a matterdominated universe from an originally matter-antimatter symmetric one. The CP-violation present in electro-weak interactions turns out to be too small to account for the present observed asymmetry by roughly eight orders of magnitude. However, physics beyond the standard model (SM), e.g., super-symmetry (SUSY), or other extensions of the SM can very well fill this void.

The physics reach of the storage ring EDM method for the proton at $10^{-29} \mathrm{e} \cdot \mathrm{cm}$ is at the $10^{3} \mathrm{TeV}$ level, much higher than the reach of the Large Hadron Collider (LHC) at CERN located in Geneva, Switzerland. The LHC looks for physics beyond the SM in a direct way with a mass reach of up to $1 \mathrm{TeV}$ or double that value when high luminosity becomes a reality. Other experimental efforts using ultra-cold neutron sources (UCN) such as the neutron EDM at the spallation neutron source (SNS) at Oak Ridge, USA, and the experiment at the Paul Scherer Institute (PSI) in Zurich, Switzerland are all suffering from statistical limitations in obtaining enough UCN intensity. Nonetheless, they are very promising experiments since they are currently setting the limits on SUSY and when operational they might discover a non-zero EDM value.

\subsection{Spin and EDM of fundamental particles}

Particles with spin $\vec{s}$ have a magnetic dipole moment given by

$$
\vec{\mu}=g \frac{q}{2 m} \vec{s}
$$

with the gyromagnetic ratio $g=2$ for point-like particles in a world without quantum fluctuations. For leptons, e.g.,

\footnotetext{
${ }^{\mathrm{a}} \mathrm{e}$-mail: yannis@kaist.ac.kr, for the Storage ring EDM collaboration
}

electrons and muons, the $g$-factor is very close to 2 and the difference, or the anomalous magnetic moment as it is called $a=(g-2) / 2 \approx 10^{-3}$, being due to quantum field fluctuations. For particles with internal structure, e.g., hadrons, the $g$-factor differs wildly from the ideal value of 2 , with the anomalous magnetic moment represented by $G=(g-2) / 2$ and of order \pm 1 , with the proton value $G_{p}=1.79$ and the deuteron $G_{d}=-0.143$. There are no particles with an anomalous magnetic moment equal to exactly zero, which is unfortunate as this would be a chance to design a very sensitive EDM experiment. Nonetheless, we can recreate the conditions where a particle will effectively behave as if it has a zero anomalous magnetic moment and we can design a very sensitive experiment. In this paper we will present such a case for the proton in an all-electric storage ring.

The particle electric dipole moment is given by

$$
\vec{d}=\eta \frac{q}{2 m c} \vec{s}
$$

with $\eta$ playing the same role as the $g$-factor in the magnetic dipole moment case.

The interaction Hamiltonian of magnetic and electric dipole moments at rest in magnetic $\vec{B}$ and electric $\vec{E}$ fields is given by

$$
H=-\vec{\mu} \cdot \vec{B}-\vec{d} \cdot \vec{E}
$$

which after applying the parity $(\mathrm{P})$ operator becomes

$$
H=-\vec{\mu} \cdot \vec{B}-\vec{d} \cdot(-\vec{E})=-\vec{\mu} \cdot \vec{B}+\vec{d} \cdot \vec{E}
$$

or the time $(\mathrm{T})$ operator

$$
H=-(-\vec{\mu}) \cdot(-\vec{B})-(-\vec{d}) \cdot \vec{E}=-\vec{\mu} \cdot \vec{B}+\vec{d} \cdot \vec{E}
$$

or the charge $(\mathrm{C})$ operator

$$
H=-(-\vec{\mu}) \cdot(-\vec{B})-(-\vec{d}) \cdot(-\vec{E})=-\vec{\mu} \cdot \vec{B}-\vec{d} \cdot \vec{E}
$$

implying that either the electric dipole moment is zero or it violates both the $\mathrm{P}$ and $\mathrm{T}$ symmetries, and under the $\mathrm{CPT}$ conservation assumption, it also violates CP-symmetry. 


\subsection{EDM sensitivity level}

In 1967, after the discovery of CP-violation in weak interactions in 1964[1] at Brookhaven National Laboratory, Sakharov published his paper[2] on the conditions required to create a matter dominated universe from an originally symmetric one after the big bang:

\section{Baryon number violation}

\section{2. $\mathrm{C}$ and $\mathrm{CP}$-violation}

\section{Interactions out of thermal equilibrium}

The current measurement of the ratio of baryons to photons is $n_{B} / n_{\gamma} \approx(6.08 \pm 0.14) \times 10^{-10}$, whereas the amount permitted by the SM through baryogenesis is only $n_{B} / n_{\gamma} \approx$ $10^{-18}$ indicating the existence of a new, much stronger source of CP-violation. An alternative process based on leptogenesis involving neutrinos at the earlier stages of the big bang could also provide a viable solution, but here we will consider only baryogenesis.

It turns out that due to the particular structure in the $\mathrm{SM}$ the weak interactions, with only one CP-violating phase, only enters at high order quantum loops as a contribution to EDMs and their values can only be $<10^{-31} e \cdot \mathrm{cm}$ for hadrons, $<10^{-38} e \cdot \mathrm{cm}$ for the electrons and $<10^{-36} e$. $\mathrm{cm}$ for the muons. Physics beyond the SM, e.g., SUSY with more than $40 \mathrm{CP}$-violating phases, contributes much more abundantly to EDMs and their contribution could be in the neighborhood of the current experimental limits. For the neutron[3] the experimental limit is $3 \times 10^{-26} e \cdot \mathrm{cm}$. Since EDMs require a chirality flip, the contribution to EDM depends to first order on the mass of the particles, i.e., $d_{e}: d_{q}: d_{\mu}=1: 20: 200$, with different, higher mass dependence also possible.

It is not true that the only contribution to the SM comes from the weak interactions $\mathrm{CP}$-violating phase. Another potential source with a much stronger EDM contribution is the so-called $\theta$-term in the QCD Lagrangian:

$$
L_{\mathrm{QCD}}=\bar{\theta} \frac{\alpha_{s}}{8 \pi} G \bar{G}
$$

with $G$ the gluon field and $\bar{\theta}$ an angle with a value of order one. From dimensional analysis the expected neutron EDM value is

$$
d_{n}(\bar{\theta}) \approx \bar{\theta} \frac{q}{m_{n}} \frac{m_{*}}{\Lambda_{\mathrm{QCD}}} \approx \bar{\theta} \cdot\left(6 \times 10^{-17}\right) e \cdot \mathrm{cm}
$$

with $m_{*}=\frac{m_{u} m_{d}}{m_{u}+m_{d}}$ the reduced up and down quark mass. When done more carefully[4] it becomes

$$
d_{n}(\bar{\theta}) \approx-d_{p}(\bar{\theta}) \approx \bar{\theta} \cdot\left(3.6 \times 10^{-16}\right) e \cdot \mathrm{cm}
$$

With $\bar{\theta}$ an angle of order one, it is expected that the neutron and proton EDMs should be of order of $3.6 \times$ $10^{-16} e \cdot \mathrm{cm}$, several orders of magnitude larger than the current experimental limit on the neutron EDM. It implies that the value of $\bar{\theta} \leq 2 \times 10^{-10}$, thus prompting Peccei and Quinn to invoke a new dynamic mechanism[5] that reduces the value of $\bar{\theta}$ below the current experimental

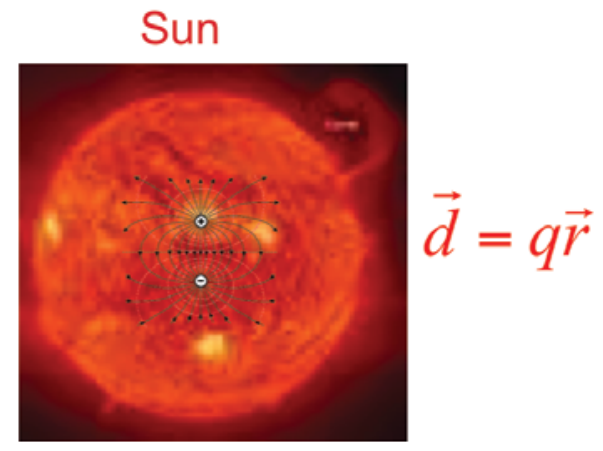

Figure 1. Expanding the proton (approximately $1 \mathrm{fm}$ in diameter) to the size of our sun (approximately 1 million km in diameter) would still require to resolve a separation of the quantum of charges by a mere $0.1 \mu \mathrm{m}$, demonstrating the sensitivity of the method.

limit. Due to breakdown of that symmetry, Weinberg[6] and Wilczek[7] suggested that there should be an axion particle present in vacuum due to the oscillating $\bar{\theta}$ around its average value of very close to zero. The oscillation frequency determines the axion mass, but this mechanism has yet to be detected.

However, the value of $\bar{\theta}$ in principle can be anything and indeed small but finite, so it can contribute to the neutron and proton EDMs at the present levels of current experimental limits. Should the neutron EDM be found to be non-zero we would want to find out the proton EDM. If the value has the same sign then it is unlikely to be due to $\bar{\theta}$, but most likely due to new physics like SUSY. Information from the deuteron is also invaluable. The deuteron, as it has a negative anomalous magnetic moment it requires a combination of magnetic and electric fields for a sensitive EDM experiment and is under development at Jülich, Germany by the JEDI collaboration[8].

It has been estimated[9] that a proton EDM with $d_{p}<$ $10^{-29} e \cdot \mathrm{cm}$ has a physics reach of

$$
d_{p} \approx 0.01\left(m_{p} / \Lambda\right)^{2} \tan \phi^{\mathrm{NP}} q / 2 m_{p},
$$

which after substituting for the parameter values

$$
d_{p} \approx 10^{-22}\left(1 \mathrm{TeV} / \Lambda_{\mathrm{NP}}\right)^{2} \tan \phi^{\mathrm{NP}} e \cdot \mathrm{cm} .
$$

If $\phi^{\mathrm{NP}}$ is of order 1 , then $\Lambda_{\mathrm{NP}} \approx 3000 \mathrm{TeV}$ is probed, while if $\Lambda_{\mathrm{NP}} \approx 1 \mathrm{TeV}$, then $\phi^{\mathrm{NP}} \approx 10^{-7} \mathrm{rad}$ is probed, demonstrating the exquisite potential sensitivity of the method.

For equal but opposite charges $\pm q$, separated by a distance $\vec{r}$ the EDM is classically defined as $\vec{d}=q \vec{r}$. If we take the proton, which has an approximate diameter of $1 \mathrm{fm}$, and blow it up to the size of our sun, with approximately diameter of 1 million $\mathrm{km}$, an EDM $d=10^{-29} e \cdot \mathrm{cm}$ means that the charge separation would be of order of $0.1 \mu \mathrm{m}$, see Figure 1.

\section{Experimental method}

It is known that electric dipole moments couple to electric fields and magnetic dipole moments to magnetic fields, 


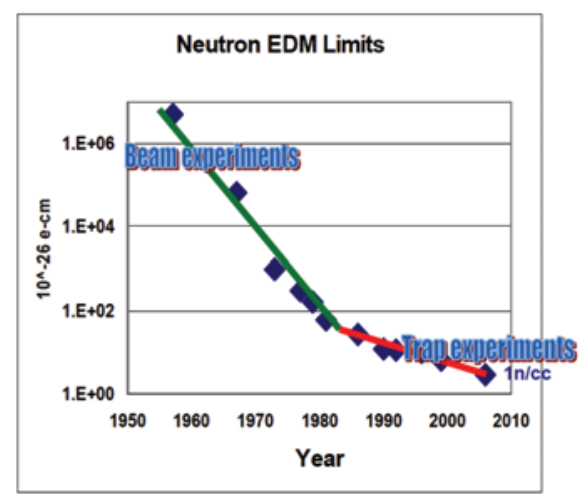

Figure 2. The neutron EDM as a function of publication year, from[10]. There is a clear kink in the EDM sensitivity owing to the potential systematic error due to motional magnetic field when neutrons move in an electric field region.

thus in order to probe the electric dipole moment of a fundamental particle the particle has to feel the presence of an electric field, which when stationary can only happen by applying an electric field to it. The spin precession rate due to this electric field is given by

$$
\frac{d \vec{s}}{d t}=\vec{d} \times \vec{E} \Rightarrow \frac{d \theta}{d t}=\frac{2 d E}{\hbar} \Rightarrow \omega=3 \mathrm{nrad} / \mathrm{s}
$$

assuming $d=10^{-29} e \cdot \mathrm{cm}$ and $E=10 \mathrm{MV} / \mathrm{m}$. The spin precession rate is very small and a large electric field is required. Ramsey and Parcel decided to use neutrons instead of charged particles in order to avoid the complications due to the application of the electric field. This method required the application of a small magnetic field so that the neutron spin precession could be observed with high sensitivity. Then a large as possible collinear electric field is applied and the spin precession rate is compared to that when the electric field direction is flipped. Since the signal is compatible with zero it is a small phase shift in the precession frequency that is mostly hoped for, i.e., the observed quantity is a scalar. To gain statistical sensitivity a large number of energetic neutrons are guided to pass between two electrically charged plates where there is a strong electric field present. The electric field direction is flipped periodically and the sought signal is a spin precession phase shift correlated with the electric field direction. This method, which had adequate statistics, ran into systematic errors and had to be abandoned for UCN traps, which unfortunately have very limited statistical sensitivity, see Figure 2. The main reason for abandoning the neutron beam experiments was due to the fact that moving neutrons in strong electric fields also feel the presence of significant magnetic fields in their rest frame, depending on their velocity, precessing their spin. When the electric field direction is flipped the neutron velocity distribution could not be controlled with the required accuracy and the method was limited by systematic errors. The storage ring proton EDM experimental method, however, makes use of the motional magnetic field and instead of a systematic error this becomes a feature of the experiment.

\subsection{Precession in a storage ring}

The spin precession rate of a particle at rest in the presence of magnetic and electric fields is given by

$$
\frac{d \vec{s}}{d t}=\vec{\mu} \times \vec{B}+\vec{d} \times \vec{E}
$$

For particles in motion the corresponding equation becomes

$$
\left(\frac{d \vec{s}}{d t}\right)^{*}=\vec{\mu} \times \vec{B}^{*}+\vec{d} \times \vec{E}^{*}
$$

where the rest frame spin precession needs to be transferred to the rest frame by taking into account the accelerated frame due to a possible particle rotation and consequently radial acceleration (Thomas precession). For a particle stored in a circular storage ring the relative spin precession compared to the momentum precession is given by (assuming $\vec{\beta} \cdot \vec{B}=\vec{\beta} \cdot \vec{E}=0$ ):

$$
\vec{\omega}_{a}=-\frac{q}{m}\left(a \vec{B}-\left[a-\left(\frac{m c}{p}\right)^{2}\right] \frac{\vec{\beta} \times \vec{E}}{c}\right)
$$

which simplifies to

$$
\vec{\omega}_{a}=-a \frac{q}{m} \vec{B}
$$

for $p=m c / \sqrt{a}$, the so-called "magic" momentum, making it independent of the electric field value. This is indeed the principle of the muon $g-2$ experiment used at CERN[11], BNL[12], and next at FNAL[13], where a uniform magnetic field is used to probe the anomalous magnetic moment of the muon with high accuracy. An electric quadrupole field is used to provide vertical focusing without affecting (to first order) $\vec{\omega}_{a}$, see Figure 3.

In the presence of an EDM the total spin precession rate is given by

$$
\vec{\omega}=-\frac{q}{m}\left[a \vec{B}+\frac{\eta}{2}(\vec{\beta} \times \vec{B})\right]=\vec{\omega}_{a}+\vec{\omega}_{\mathrm{edm}}
$$

causing a tilt in the total angular precession vector $\vec{\omega}$ with respect to the vertical, with $\tan \theta=\omega_{\text {edm }} / \omega_{a}$, see Figure 4. In general the sensitivity of the experiment to the muon EDM is limited[15] especially compared to the electron EDM limit[16] due to the finite muon lifetime, the weak muon beam intensities and the diffused muon phase space available. However, the muons are the only second generation particle that we have a chance of reaching better than roughly $10^{-21} e \cdot \mathrm{cm}$ in the FNAL[13] and JPARC [14] experiments in an indirect way. It is possible to improve that sensitivity, especially the systematic errors, by a couple more orders of magnitude in a dedicated storage ring experiment by using a combination of electric and magnetic fields to freeze the muon spin in the horizontal plane[17, 18].

\subsection{Storage ring proton EDM}

The dedicated storage ring proton EDM experiment based on a dedicated electric field storage ring is the simplest 


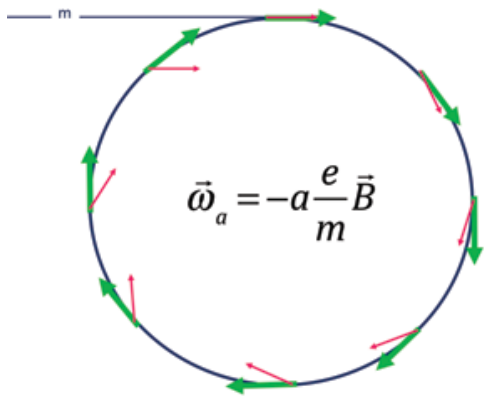

Figure 3. The muon spin precession slightly faster than the muon momentum in a uniform magnetic field ring with electric focusing and the muons at their magic momentum.

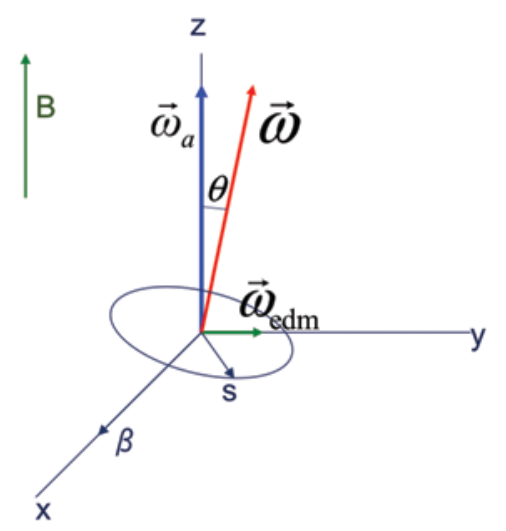

Figure 4. The total muon spin precession plane is slightly tilted from the vertical due to the small contribution by the muon EDM.

way to accomplish high sensitivity in hadronic EDMs. High intensity (more than $10^{11}$ per cycle) with high beam polarization (more than $80 \%$ ) are readily available at several labs around the globe, e.g., BNL in the USA and COSY in Jülich/Germany. The anomalous magnetic moment of the proton is $G=1.79$ and the magic momentum becomes $p=700 \mathrm{MeV} / \mathrm{c}$. The spin and momentum precession rate coincide at the magic momentum value with the radial E-field being always on the same side of the EDM vector and the EDM signal is the spin precession into the vertical plane, acting for the duration of the storage time, see Figure 5. The EDM signal causes a spin precession in the vertical plane, perpendicular to the magnetic dipole moment precession plane in the more familiar $g-2$ case and can therefore be distinguished.

An all electric ring with a radius of $R=40 \mathrm{M}$ requires a radial E-field of $E \approx 10 \mathrm{MV} / \mathrm{m}$ and can be accomplished at a reasonable cost. In a realistic lattice there is a need for beam injection as well as beam diagnostics. In an allelectric ring, counter-rotating beams can be stored simultaneously. This feature is critically important since the main systematic error source that is a non-zero radial B-field, will split the counter-rotating beams vertically. The vertical separation depends on the strength of the vertical focusing, encouraging a rather weak vertical focusing. This



Figure 5. The proton spin follows the momentum exactly, on average, in the horizontal plane $\left(\vec{\omega}_{a}=0\right)$, while the EDM signal $(d \vec{s} / d t=\vec{d} \times \vec{E})$ accumulates for the duration of the storage time causing the spin to precess out of plane.

vertical separation creates a radial B-field, which is modulated at $1-10 \mathrm{kHz}$ by modulating the strength of the vertical focusing by $1-10 \%$. A realistic (to first order) ring lattice is given in Figure 6. The distortion of the closed orbit in the vertical direction for a single beam is given by

$$
y(\theta)=\sum_{N=0}^{\infty} \frac{\beta c R_{0} B_{r, N}}{E_{0}\left(Q_{y}^{2}-N^{2}\right)} \cos \left(N \theta+\phi_{N}\right)
$$

where $E_{0}$ is the radial E-field strength, $Q_{y}=$ $Q_{y 0}\left[1+A_{m} \times \cos \left(\omega_{m} t+\phi_{m}\right)\right]$ is the modulated vertical tune, and $N$ is the radial B-field harmonic around the ring azimuth.

Only the $N=0$ radial B-field harmonic is a systematic error source. However, the counter-rotating beams may sense a non-zero effect originating from a higher harmonic and could be a potential systematic error. The ratio of the strength of a non-zero $N^{\text {th }}$ harmonic to the $N=0$ case for the time dependent part is proportional to $\left(Q_{y} / N\right)^{4}$, which for small $Q_{y}$, e.g. $Q_{y}=0.3$ and somewhat large value for $N$, e.g., $N=10$, the effect diminishes by six orders of magnitude. Therefore, if the SQUID-based BPMs are sensitive up to the $10^{\text {th }}$ harmonic of the radial B-field, reducing the field everywhere below $0.1 \mathrm{nT}$ level is adequate. The oscillating radial magnetic field is sensed using SQUID-based magnetometers with single sensor sensitivity of about $3 f T / \sqrt{H z}$, much better than needed for $10^{-29} e \cdot \mathrm{cm}$ overall sensitivity. The requirement is to have approximately one dozen SQUID-based beam position monitors (BPMs) around the ring, with the BPM segments of the ring to be at cryogenic temperatures of about 4 K, shown in Figure 7.

Another potential systematic error is the geometrical phase, again related to magnetic fields and the ring plate alignment. It turns out, by using high precision beam/spin dynamics simulations[19-21], that in an all-electric storage ring those systematic errors are easier to handle than in tradition trap experiments. The counter-rotating beams can be sensed separately and the traditional BPMs provide enough information to control this error below the statistical sensitivity as long as the absolute level of the B-fields is less than $0.5 \mathrm{nT}$ and the magnetic field gradient is below $0.1 \mathrm{nT} / \mathrm{m}$. These requirements ordinarily would require an 


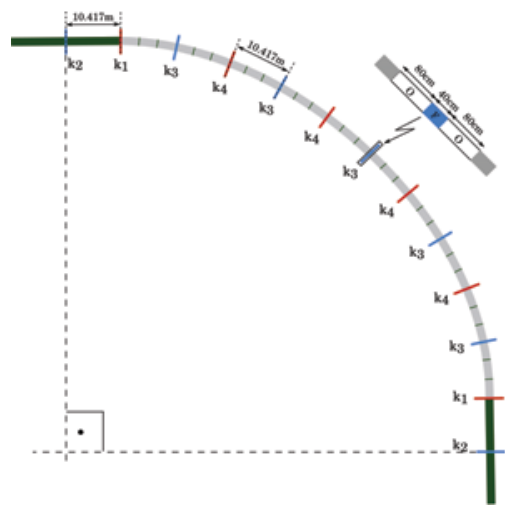

Figure 6. The proton ring lattice has several straight sections accommodating injection sections, polarimeter sections and SQUID-based beam position sections (see text). Alternate focusing is used with the horizontal being as strong as possible to increase the ring admittance and the vertical being rather weak to increase sensitivity to the radial B-field. The radial B-field splits the counter-rotating beams depending on the vertical focusing strength.

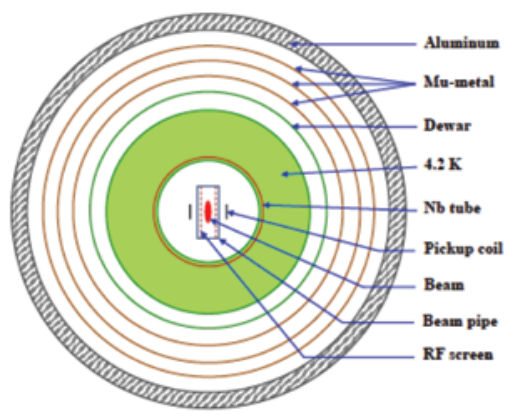

Figure 7. A net radial B-field in the ring would be a systematic error. However, it also splits the counter-rotating beams which depends on the strength of the vertical focusing. The beam splitting creates a radial $\mathrm{B}$-field, which we modulate by modulating the vertical focusing at 1-10 kHz making it possible to be sensed using high sensitivity SQUID-based magnetometers.

elaborate mu-metal shielding around the ring, which could be very expensive. However, recent developments[22] allow for an affordable mu-metal shielding scheme by applying a new degaussing (or equilibration as it is now called) technique. Figure 8 shows a cut-out of the two layer shielding that is deemed to be sufficient to achieve the required shielding.

The statistical sensitivity of the experiment is given by

$$
\sigma_{d}=\frac{2 \hbar}{E_{R} P A \sqrt{N_{c} f \tau_{p} T_{\mathrm{tot}}}}
$$

with $P=0.8$ the beam initial polarization, $\tau_{p}=10^{3} \mathrm{~s}$ the beam polarization lifetime, $A=0.6$ the average left/right asymmetry observed by the polarimeter, $N_{c}=10^{11}$ the total number of particles stored per cycle, $T_{\text {tot }}=10^{7} \mathrm{~s}$ the total running time per year, $f=1 \%$ the total useful event rate fraction, and $E_{R}=7 \mathrm{MV} / \mathrm{m}$ the average radial electric

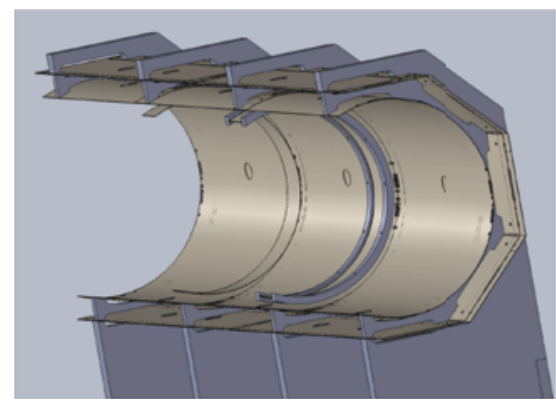

Figure 8. A two layer shielding with special equilibration coils is expected to provide adequate shielding (see text). A prototype of the shielding has been purchased by the Technical University of Munich and is under testing at CAPP/IBS in the KAIST campus.

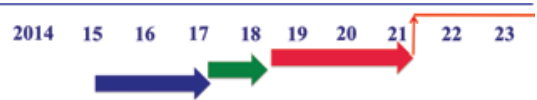

Figure 9. A technically driven schedule is shown here. At CAPP/IBS we are well on our way with the final R\&D items required to obtain the necessary information for the final ring optimization. After that time we should be able to finish the ring design in one year and by 2021 we could complete the ring construction.

field around the ring. As a result, the estimated $\sigma_{d}=1 \times$ $10^{-29} e \cdot \mathrm{cm} /$ year.

\subsection{Plan for the storage ring EDM method}

A good EDM experiment should have good statistical sensitivity:

1. High intensity beams

2. Long beam lifetime

3. Long spin coherence time

and in addition it needs to have a good way of canceling major systematic errors:

1. Control or cancel the magnetic field effects

2. Small sensitivity to geometrical phase effects and ways to cancel them

\section{Detector related systematic errors}

4. Negligible dark current from the electric field plates

The proton EDM method has all those stated features and indeed the collaboration is confident that it can put together a successful team to build it and run it within a reasonable cost and schedule. The rough schedule as outlined at the present time is shown in Figure 9 


\subsection{A storage ring electron EDM: Does it make sense?}

The magic momentum of the electron is only $15 \mathrm{MeV} / \mathrm{c}$, making it an ideal candidate for a demonstration storage ring EDM experiment and indeed a sensitive probe for a direct measurement of the electron EDM. Yuri Orlov first pointed this out around 2004 at which time the storage ring EDM collaboration tried to find a sensitive and efficient electron polarimeter for it. It turns out that this is a very difficult electron energy and at the time there was nothing available that could be used for an experiment. William Morse tried to resurrect the idea in 2013 after we came up with the all-electric storage ring idea for the proton and the simultaneous counter-rotating beams. Recently, in 2015 Richard Talman published a new idea[23] where by modifying the observational principle of the experiment and by developing a super-conducting resonance technique one may indeed be possible to put together a sensitive electron EDM experiment.

It is worthwhile to investigate these new options and come to a conclusion whether or not it makes sense to proceed. Even in the worst case, such a ring will become a testing ground for all the relevant, high risk items of the proton EDM experiment and it's probably a good idea to proceed.

\subsection{Conclusions and acknowledgements}

The current status in physics is very intriguing and indeed puzzling. The available phase-space for SUSY is closing in and it would be nice to have the option of building a machine with much larger energy reach. This may materialize some day, but it's going to take at least a decade to develop even if we had the funding. Storage ring EDM experiments with their very high New Physics mass scale reach can fill the void and provide guidance for the next HEP machine.

I am grateful to my colleagues in the storage ring EDM collaboration for all their ingenuity and hard work over the years and to Octavio Escalante for reading the document very carefully and for his excellent comments. This work was supported in part by IBS of South Korea (IBS-R017D1-2016-a00).

\section{References}

[1] J.H. Christenson, J.W. Cronin, V.L. Fitch, and R. Turley, Phys. Rev. Lett. 13, 138 (1964)

[2] A.D. Sakharov, JETP Lett. 5, 24 (1967)

[3] C.A. Baker et al., Phys. Rev. Lett. 97, 131801 (2006)

[4] M. Pospelov, A. Ritz, Ann. Phys. 318, 119 (2005)

[5] R.D. Peccei and H.R. Quinn, Phys. Rev. Lett. 38, 1440 (1977)

[6] S. Weinberg, Phys. Rev. Lett. 40, 223 (1978)

[7] F. Wilczek, Phys. Rev. Lett. 40, 279 (1978)

[8] JEDI collaboration, http://collaborations.fz-juelich. de/ikp/jedi/collaboration/collaboration.shtml

[9] W. Marciano, private communication

[10] W. Morse, private communication

[11] J. Bailey et al., Nucl. Phys. B150, 1-75 (1979)

[12] G. Bennett et al., (Muon G-2 Collaboration), Phys. Rev. D73, 072003 (2006), hep-ex/0602035

[13] J. Grange et al., Muon $(g-2)$ Technical Design Report: FERMILAB-FN-0992-E

[14] N. Saito and J-PARC $(g-2)$ /EDM Collaboration, AIP Conf. Proc. 1467 (2012) 45. doi: 10.1063/1.4742078

[15] G.W. Bennett et al., Phys. Rev. D80, 052008 (2009)

[16] J. Baron et al., Science 343269 (2014)

[17] F.J.M. Farley et al., Phys. Rev. Lett. 93052001 (2004)

[18] A. Silenko et al., J-PARC Letter of Intent: Search for a Permanent Muon Electric Dipole Moment at the $10^{-24} e \cdot \mathrm{cm}$ Level, Jan. 2003

[19] V. Anastassopoulos et al., "A Storage Ring Experiment to Detect a Proton Electric Dipole Moment", arXiv:1502.04317, 2015

[20] E.M. Metodiev et al., Phys. Rev. ST Accel. Beams 17 (2014) 7, 074002

[21] E.M. Metodiev et al., Nucl. Instrum. Meth. A797 311 (2015)

[22] I. Altarev et al., J. Appl. Phys. 117233903 (2015). doi: 10.1063/1.4922671

[23] R. Talman, "Frequency Domain Storage Ring Method for Electric Dipole Moment Measurement", arXiv:1508.04366, 2015 